\title{
The Implementation of DCGAN in the Data Augmentation for the Sperm Morphology Datasets
}

\author{
Kamran Balayev $^{1}$, Nihad Guluzade ${ }^{1}$, Sercan Aygun ${ }^{1 *}$, Hamza Osman Ilhan ${ }^{1}$ \\ 1 Yildiz Technical University, Faculty of Electrical-Electronics, Department of Computer Engineering, Istanbul, Turkey, \\ (ORCID: 0000-0002-0056-8152), 11117904@std.yildiz.edu.tr \\ (ORCID: 0000-0003-0482-2303), 11117903@std.yildiz.edu.tr \\ (ORCID: 0000-0002-4615-7914), ayguns@yildiz.edu.tr \\ (ORCID: 0000-0002-1753-2703), hoilhan@yildiz.edu.tr
}

(3rd International Congress on Human-Computer Interaction, Optimization and Robotic Applications June 11-13, 2021)

(DOI: $10.31590 /$ ejosat.952561)

ATIF/REFERENCE: Balayev, K., Guluzade, N., Aygun, S. \& Ilhan, H. O. (2021). The Implementation of DCGAN in the Data Augmentation for the Sperm Morphology Datasets. European Journal of Science and Technology, (26), 307-314.

\begin{abstract}
A large amount of data is the key requirement in order to train a neural network efficiently. Using a small size training set in network training causes low accuracy for model performance over the testing set and also hard to implement the model in practice. Similar to many other problems, sperm morphology datasets are also limited for training the neural network-based deep networks in order to provide an automatic evaluation of sperm morphometry. Data augmentation mitigates this problem by utilizing actual data more effectively. The standard data augmentation techniques focus on only spatial changes over the images and can only produce a restricted number of useful informative and disjunctive data. Therefore, in order to create more distinctive and diverse data than the regular spatial domain-based augmentation techniques, a deep learning-based data augmentation technique which is known as the generative model, is trained in this study for the sperm morphology datasets. The deep convolutional generative adversarial network (DCGAN) was optimized and utilized in this study for three well-known sperm morphometry datasets as SMIDS, HuSHeM, and SCIAN-Morpho. Each dataset was individually augmented to a 1000 sample size by the proposed approach. In order to optimize the network with different parameters and observe the generated data, a graphical user interface has been designed. For the similarity evaluation of the generated images to original images, the Fréchet Inception Distance (FID) score was utilized. The FID results indicate that the most similar generated images have been obtained for SMIDS with an average of 29.06 FID score. The worst performance (Average FID = 53.46) was obtained for the SCIAN-Morpho dataset, which has low resolution and data imbalance problems. Lastly, DCGAN based proposed approach resulted in an average of 44.25 FID score for the HuSHeM dataset.
\end{abstract}

Keywords: Data Augmentation, DCGAN, Deep Learning, Generative Adversarial Networks, Sperm Morphology.

\section{Sperm Morfolojisi Veri Kümeleri için Veri Artırmada DCGAN'ın Uygulanması}

$\ddot{\mathbf{O z}}$

Bir sinir ağını verimli bir şekilde eğitmek için büyük miktarda veri temel gereksinimdir. A $\breve{g}$ eğitiminde küçük boyutlu bir eğitim kümesinin kullanılması, test kümesinde düşük doğruluklu model performansına neden olur ve modelin pratikte uygulanmasını zorlaştırır. Diğer birçok soruna benzer şekilde, sperm morfolojisi veri kümeleri de, sperm morfometrisinin otomatik

\footnotetext{
* Corresponding Author: Yildiz Technical University, Faculty of Electrical-Electronics, Department of Computer Engineering, Istanbul, Turkey, ORCID: 0000-0002-4615-7914, ayguns @ yildiz.edu.tr
} 
değerlendirilmesini sağlamak için sinir ağı tabanlı derin ağları eğitmek açısından sınırlıdır. Veri artırma, gerçek verileri daha verimli kullanarak bu sorunu azaltır. Standart veri artırma teknikleri, yalnızca görüntüler üzerindeki uzamsal değişikliklere odaklanır ve sadece sınırlı sayıda yararlı bilgi sağlayan ve ayrık veri üretebilir. Bu nedenle, standart uzamsal tabanlı veri artırma tekniklerinden daha farklı ve çeşitli veriler oluşturmak için, bu çalışmada sperm morfolojisi veri kümeleri adına üretici model olarak bilinen derin öğrenme tabanlı bir veri artırma tekniği kullanılmıştır. Bu çalışmada Derin Evrişimli Üretici Çekişmeli Ağ (DCGAN) optimize edilmiş ve SMIDS, HuSHeM ve SCIAN-Morpho gibi üç iyi bilinen sperm morfometrisi veri kümeleri kullanılmıştır. Her veri kümesi, önerilen yaklaşımla ayrı ayrı 1000 örneklem büyüklügüne yükseltilmiştir. Ağı farklı parametrelerle optimize etmek ve üretilen verileri gözlemlemek için grafiksel bir kullanıcı arayüzü tasarlanmıştır. Üretilen görüntülerin orijinal görüntülere benzerlik değerlendirmesi için Fréchet Başlangıç Mesafesi (FID) skoru kullanılmıştır. FID sonuçları, oluşturulan en benzer görüntülerin ortalama 29.06 FID puanı ile SMIDS için elde edildiğini göstermektedir. En kötü performans (Ortalama FID = 53.46), düşük çözünürlük ve veri dengesizliği sorunları olan SCIAN-Morpho veri kümesi için elde edilmiştir. Son olarak, DCGAN tabanlı önerilen yaklaşım HuSHeM veri kümesi için ortalama 44.25 FID puanıyla sonuçlanmıştır.

Anahtar Kelimeler: Veri Artırma, DCGAN, Derin Öğrenme, Üretici Çekişmeli Ağlar, Sperm Morfolojisi.

\section{Introduction}

In deep learning-based classification approaches, the performances largely depend on the amount of data available for training. In case of using insufficient data in training causes high error rates in the practical usage of the model. Scientific efforts such as the classification of various illnesses may not easily be carried out due to sufficient data unavailability (Vasconcelos \& Vasconcelos, 2017). Similarly, one of the medical fields suffering from the limited dataset problem is that of sperm morphometry. The morphological analysis of sperm shapes provides the key information about the male factor in infertility problems. However, the data is not abundant due to privacy concerns. In the laboratories, morphological analysis has been performed by the visual assessment technique by experts who carry out manual observations under the microscope without storing the images. In this procedure, the results strongly depend on the expertise of the observer. Different observers report different results, known as the observer variability problem (Kapoor, 2021). In order to eliminate the human factor and develop a computer-based analyzing approach for the determination of the sperm shapes with more objective and consistent results, the analyzing system should be fed with a lot of labeled images in the training phase. Therefore, the data augmentation techniques are a vital preprocessing step in this field.

In the data augmentation techniques, the main idea is to create synthetic images from the existing datasets via spatial domain image processing or deep learning methods. In this study, we aim to train one of the recently growing fields of deep learning models, namely adversarial networks, for data augmentation. By combining the number of input domains using Generative Adversarial Networks (GAN) (Goodfellow et al., 2014), new data is generated; thereby, a comprehensive dataset is created. The proposed deep learning-based augmentation approach has been individually utilized over three well-known publicly available sperm morphology datasets to increase the sample sizes by generating new images. Additionally, a graphical user interface (GUI) was designed to easily optimize the network and demonstrate the generated images. The proposed GAN-based approach provides a solution, especially for the medical field where the data is scarce due to privacy and ethical concerns.

\section{Related Works}

The performance of machine learning applications, such as the classification of images, is directly related to the amount of data (Vasconcelos \& Vasconcelos, 2017). On the other hand, finding sufficient and reliable datasets are the most challenging phase. Therefore, developing synthetic data augmentation techniques in image classification tasks is very popular due to eliminating this bottleneck. Data augmentation methods can be defined as the data generation techniques by performing spatial image manipulation operations or deep learning-based methods. The generated data should be informative and also distinctive from the original image. Rotating, shifting, cropping, fading, frequency masking, scaling, etc., are several examples utilized in spatial-based image manipulation operations. These techniques provide to increase the number of training images, but the generated images are not so much different than original images. Therefore, the training process of networks may not be enriched in some applications. Using deep learning-based data augmentation techniques, the generated images are more informative and different from original images, providing a more enhanced training process. However, the fine-tuning procedure of the generative networks is the critical step that should be set carefully.

In literature, several studies were conducted to investigate the effects of spatial data augmentation techniques in the classification of sperm morphology datasets (Ilhan et al., 2020; Tortumlu \& Ilhan, 2020; Yüzkat et al., 2020). Ilhan et al. performed the spatial augmentation techniques on the SMIDS dataset in order to increase the classification performance of three deep networks. Augmentation provided 1.4\%, 3\%, and $3.3 \%$ increments for VGG19, Inception, and Mobile Net, respectively. However, they also reported that excessive training times were observed in the training of networks due to the data generation step. Additionally, some networks have resulted in lower accuracy after a point of augmentation scale because of feeding the almost similar images to networks. This is the limitation of the spatial-based augmentation techniques, which causes the non-informative training phase, as demonstrated in (Yüzkat et al., 2020). In another study, the effects of spatial augmentation techniques have been explored for mobile-based networks (Tortumlu \& Ilhan, 2020). They tested over three sperm morphology datasets and reported that the augmentation approach increased the performance for all datasets. Lastly, Yuzkat et al. focused on the performance increment for the most challenging sperm morphology dataset, SCIAN-Morpho. They tested multiple networks with several parameters and augmentation scales. Eventually, they indicated that the 
augmentation is also effective over the low-resolution image sets as well.

As deep learning-based data augmentation techniques evaluate, GANs are the most implemented techniques in recent years (Goodfellow et al., 2014). GANs have many different versions but are commonly designed based on deep convolutional networks in two ways: discriminator and generator (Barışkan et al., 2020; Radford et al., 2016). Progressive growth of GANs (Karras et al., 2018) uses a pathway that is gradually enlarged by increasing the number of layers in both generator and discriminator networks. With the flexibility of GAN networks, higher frequency details are added to the image created in the energy-based GAN model (Zhao et al., 2017) to produce effective results. In literature, GAN-based networks were implemented in different medical image datasets but not tested over Sperm Morphometry. Rubin et al. used GAN with the transfer learning idea in the label-free live cell dataset (Rubin et al., 2018). The utilized dataset also has a small size training set, resulting in low accuracy for transfer learning. They performed GAN architecture to generate more synthetic live-cell images to fed the networks. In another study of Rubin et al., they proposed a novel GAN architecture, namely TOP-GAN (transferring of pre-trained generative adversarial network). They also utilized TOP-GAN for the augmentation of a smallsize cancer image dataset. They achieved an average of $95 \%$ accuracies for a small-size cancer dataset by the TOP-GAN model (Rubin et al., 2019).

GAN models are also used in different fields. More recently, in the style-based architecture (Karras et al., 2019), instead of feeding the data used as input directly to the generator, this input is first transformed into a hidden space and used to measure and slide the standardized image characteristic output calculated from convolutional layers. Apart from data augmentation techniques, GAN networks are also applied for some complex tasks. For instance, the recently mentioned GAN model (Mirza \& Osindero, 2014) can be used for modeling the special movement of patient distribution bottomed on before surgery of image, re-colorization of endoscopic video data, and highlighting regions most affected by a disease. Another task as style transfer is a useful image synthesis technique that can re- process the given image to another artistic style while preserving the image of the content. GANs are a widely adopted framework for this task because of their better representation of local style patterns than traditional Gram-matrix-based methods. Most of the previous methods are based on pre-assembled style images to train the model. A new Patch Permutation GAN ( $\left.{ }^{2}-G A N\right)$ network (Zheng \& Liu, 2020) can effectively learn the contour style from a single style image and is used to create multiple training examples. A patch separator is designed, which can seamlessly process patch-based and natural images at the same time. In addition, a local texture descriptor-based criterion is proposed to evaluate style transfer quality quantitatively. Experimental results show that this method (Zheng \& Liu, 2020) produces a higher quality image than a single style image with improved computational efficiency compared to many modern techniques. GAN networks are also utilized for the improvement of image resolution (Ledig et al., 2017; Yeh et al., 2017; Zhang et al., 2017).

In this study, DCGAN that is one of the GAN based architecture has been implemented to generate sperm patch images in order to augment data of small training sets. The performance metrics show that the created new images are similar to original images but consist of additional information different than the images generated by the regular spatial data augmentation techniques.

\section{Materials and Methods}

\subsection{Dataset Information}

The aim of the study is to create large sperm morphology datasets for different sperm morphology image sets. In this regard, three well-known publicly available datasets, namely SMIDS, HuSHeM, and SCIAN-Morpho, are utilized for training and testing of generative models. For datasets containing a very low amount of images, we use standard spatial-based data augmentation techniques, increasing the count of images to the required level for training of GANs. The number of original images contained in each dataset is shown in Table 1. The train and test partitions of the datasets are divided using the cross-fold format.

Table 1. Class names and sample sizes in the utilized datasets

\begin{tabular}{lc|lc|lc}
\multicolumn{2}{c}{ SMIDS } & \multicolumn{2}{c}{ HuSHeM } & \multicolumn{2}{c}{ SCIAN-Morpho } \\
\hline Labels & \# of images & Labels & \# of images & Labels & \# of images \\
\hline Normal & 1021 & Normal & 54 & Normal & 100 \\
Abnormal & 1005 & Tapered & 53 & Tapered & 228 \\
Non-Sperm & 974 & Pyriform & 57 & Pyriform & 76 \\
& & Amorphous & 52 & Small & 72 \\
& & & & Amorphous & 656 \\
\hline \multirow{2}{*}{ Total } & \multirow{2}{*}{3000} & Total & 216 & Total & 1132 \\
\hline
\end{tabular}


SMIDS was obtained from the Medical Faculty of Istanbul University. A smartphone-based data acquisition approach was used in the collecting of the semen samples. This novel data acquisition approach has been introduced in (Ilhan \& Aydin, 2018) and validated over the motile sperm and concentration analysis. Totally 3000 segmented patches have been manually labeled as normal (1021), abnormal (1005), and non-sperm (974) patches by an expert. HuSHeM data set was created from semen samples of 15 patients in Isfahan Fertility and Infertility Center. It has four sub-classes which are normal, tapered, pyriform, and amorphous (Shaker et al., 2017). The images in the dataset are almost equally distributed into classes (around 50 samplers per class) but not enough to train for a deep network. Lastly, The SCIAN-Morpho consists of manually cropped and rotated human semen samples taken from the University of Chile, Faculty of Medicine Spermiogram Laboratory Anatomy and Developmental Biology Program (Chang et al., 2017). The images were collected in a very small size of resolution, $35 \times 35$, gray-scale color space, and having imbalanced distribution per class. Therefore, the SCIAN-Morpho dataset is known as the most challenging dataset in this field. The effective data augmentation technique would provide a significant increment in the classification.

\subsection{Methodology}

In the proposed study, The Deep Convolutional Generative Adversarial Network (DCGAN) has been implemented for sperm morphology image augmentation. DCGAN is one of the widely improved GAN structures used in the data augmentation steps for many other studies in the literature (Karras et al., 2019; Rubin et al., 2019). It uses the convolutional neural layers for both the generator and discriminator models. The training for this type of GAN results in more stable and accurate outcomes. This structure is used in combination with convolutional neural networks (CNNs) and GAN networks. In the structure of the model in this study, unlike other adversarial neural networks, convolutional networks are used that replace deterministic pooling functions such as max-pooling with long convolutions. This model that directly connects the largest convolutional layers of both generator and the discriminator pattern in the fundamental construction of the GAN model has shown encouraging outcomes in the latest studies. While other GAN models have used the single momentum to accelerate training, DCGAN uses Adam optimizer with tuned hyperparameters to achieve stable training results.

Three aforementioned datasets are sourced into the DCGAN architecture. For each dataset, the DCGANs were adequately trained by using different parameter determination and optimization settings. Then, 1000 images per class are generated after the training phase. The overall flow of the presented approach is depicted in Figure 1. The initial parameters, which are experimentally decided for the best performance, are given in Table 2.

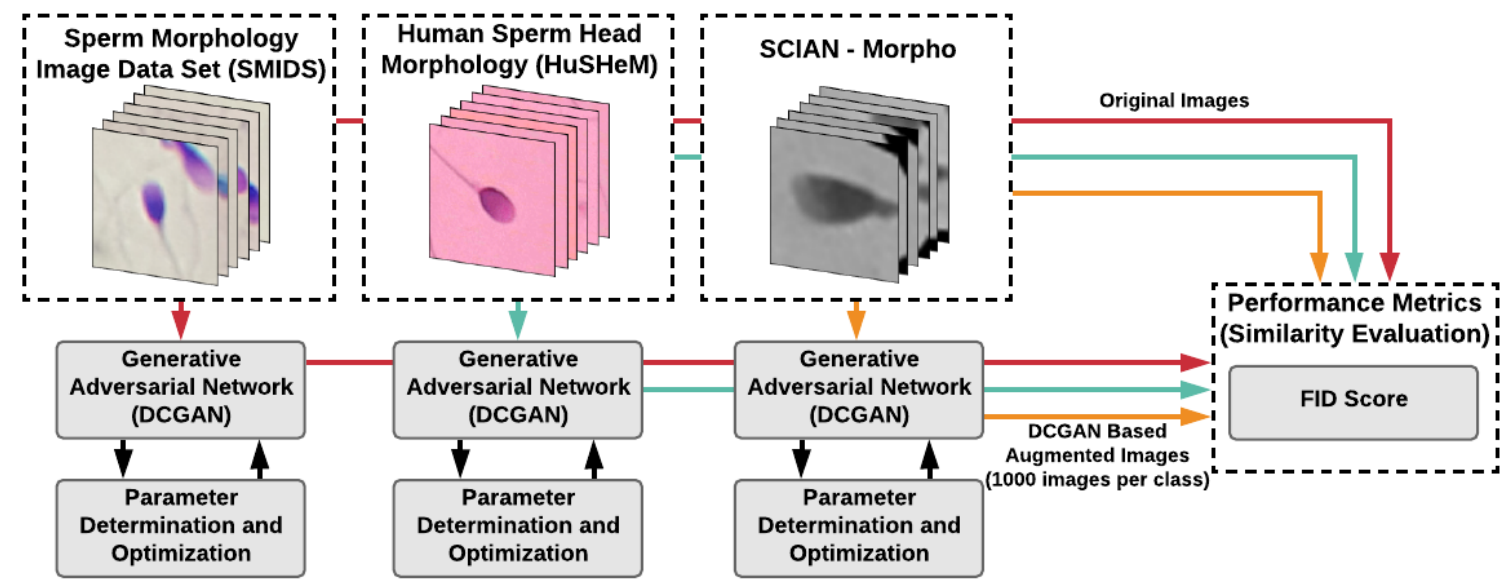

Figure 1. The flowchart of the presented approach

The activation functions in machine learning are in charge of processing the total input values from the node to the output. In other words, the activation determines whether the representations from the input domain are available in the different places of the forward path. As in real-life practices, these relationships require non-linear operations, some special mathematical functions, such as ReLU activation, are used. The following activation functions are the crucial parameters arranged in DCGAN in the proposed data augmentation approach.
1) Rectified Linear Unit: ReLU causes the model to learn more quickly during the training. ReLU has grown to be the default activation feature for many neural networks, especially $\mathrm{CNN}$, since it is an activation function that simplifies the training procedure.

2) Leaky ReLU: Leaky ReLU is an activation function that fixes the "dying ReLU" problem. This problem arises when the neurons, linked to this function, always output 0 as the negative inputs cause. 
Table 2. Parameter determination of DCGAN for each utilized sperm morphology dataset

SMIDS

HuSHeM

SCIAN-Morpho

\begin{tabular}{lc|lc|lc}
\hline Parameters & Equivalent & Parameters & Equivalent & Parameters & Equivalent \\
\hline Noise size & 128 & Noise size & 128 & Noise size & 128 \\
Learning rate for $D$ & 0.001 & Learning rate for $D$ & 0.00004 & Learning rate for $D$ & 0.00004 \\
Learning rate for $G$ & 0.001 & Learning rate for $G$ & 0.00004 & Learning rate for $G$ & 0.00004 \\
Batch size & 64 & Batch size & 64 & Batch size & 64 \\
Epochs & 2,000 & Epochs & 2,500 & Epochs & 2,000 \\
Betal & 0.9 & Betal & 0.9 & Betal & 0.9 \\
Weights & 0.002 & Weights & 0.002 & Weights & 0.002 \\
Epsilon & $1 \mathrm{e}-08$ & Epsilon & 0.005 & Epsilon & 0.005 \\
\hline
\end{tabular}

\subsection{Performance Metric}

The performance of the DCGAN based data augmentation technique, especially for the sperm morphological datasets, has been measured using Fréchet Inception Distance (FID) score. The formula of FID is given in Equation 1. FID is an objective metric that calculates the distance between feature vectors of original and generated (artificial) images. This metric is also involved in the utilization of the InceptionV3 model; deep learning neural network for image classifications. A low FID value indicates that the two image datasets are similar and having more similar close statistics.

$$
\begin{aligned}
& d^{2}\left((m, C),\left(m_{w}, C_{w}\right)\right)= \\
& \left\|m-m_{w}\right\|_{2}^{2}+\operatorname{Tr}\left(C+C_{w}-2\left(C C_{w}\right)^{1 / 2}\right)
\end{aligned}
$$

FID value is expressed as $d^{2}$, where $m$ and $m_{w}$ represent the average of real and generated images in terms of their properties.
$C$ and $C_{w}$ are the covariance matrix for real and generated feature vectors called sigma. $\operatorname{Tr}$ corresponds to the trace linear algebra operation.

\subsection{Application}

The development environment of this study was implemented in Python programming language (Python 3.6) using Keras library within TensorFlow. Since speed and efficiency are important factors, PyCharm Community application development environments provided by Google Colab and JetBrains were mainly used. The GUI for the bulk image generation using the backend framework of the overall neural network structure was designed as shown in Figure 2. It was preferred to use the system interface with the Tkinter tool that comes with the Python programming language on the front side.

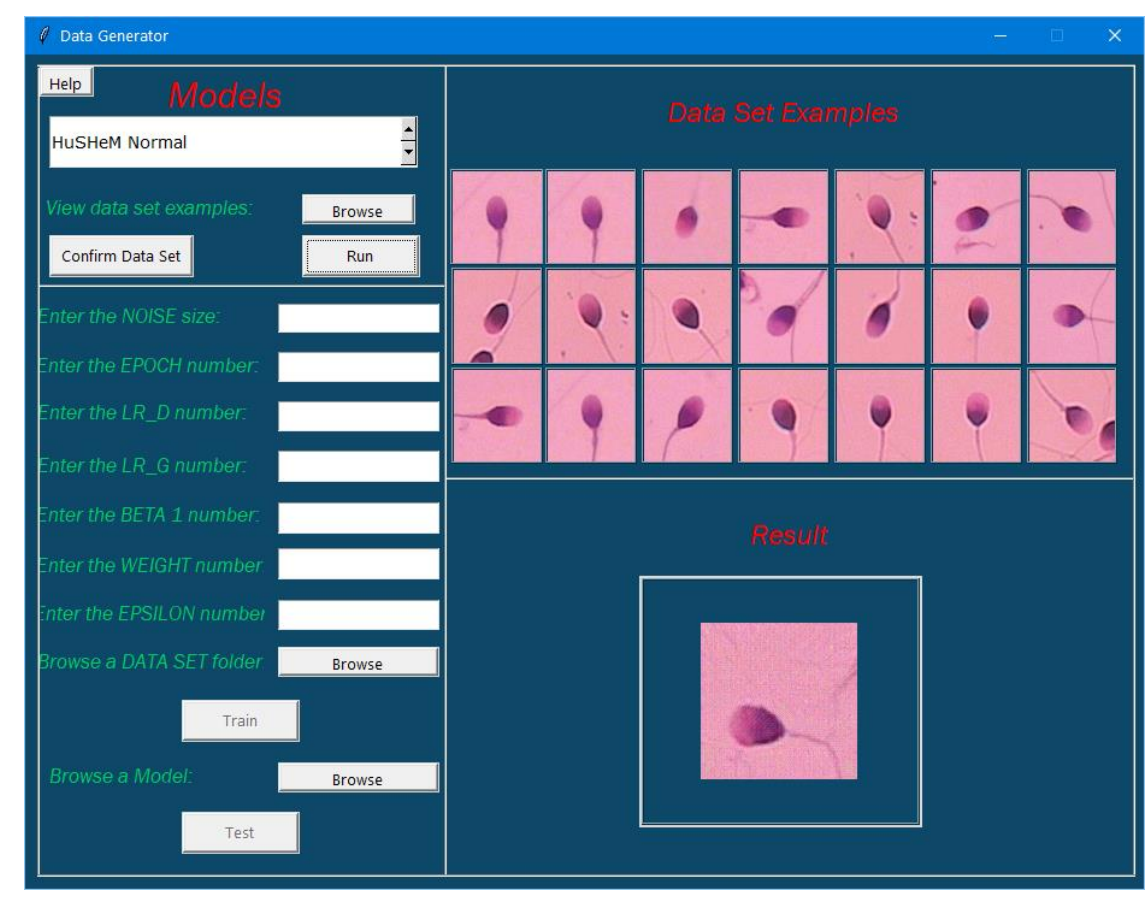

Figure 2: GUI of the application 
The main idea of developing a GUI is to ease the process of fine-tuning for DCGAN. Users can easily arrange the parameters by using the GUI and start the training process. Additionally, GUI has been designed, dataset independent. Therefore, GUI can be used for any dataset to be augmented, not just for Sperm Morphology Datasets. The application is open source and freely downloadable from (Balayev et al., 2020).

\section{Experimental Results}

In the experimental phase of our research, different scenarios and parameter values have been tested to ensure a completely realistic synthetic version of images. We have the generated images as all the features from the original input domain. It was seen that values of the most appropriate results during the trials changed according to the size and characteristics of the applied datasets. In every machine learning system, statistical information about the success of the system is required by using different methods on the available data in order to measure the success of the installed system, thereby obtaining information about the accuracy and consistency. While the evaluations of the images were created at the initial stages, the testing was primarily conducted via human visual perception. Then fine-tuning is achieved according to the FID score metrics.

Early trials of the images were conducted on the SMIDS dataset with the default parameter values taken from the original paper of the DCGAN model (Radford et al., 2016). However, these parameters did not perform promisingly for the HuSHeM and SCIAN-Morpho types of sperm datasets as the high learning rate caused the mode collapse problem, frequently observed on adversarial networks. We observed that, while the constant low learning rate of the networks used in training increased the overall quality of images by making the training more stable, it significantly reduces the time of the training process. We assigned learning rates for HuSHeM and SCIAN-Morpho datasets by logarithmically increasing and decreasing fashion. For the evaluation of the study, we kept the high noise size for all types of datasets to create more diverse data. Several examples for the generated samples are presented in Table 3. We compared our generated results with the original input dataset, and we observed that the artificial results resemble the original input domain quite adequately.

At the end of the study, FID scores were calculated for the generated results and given in Figure 3 using the bar plots. Low scores correspond to how synthetic images are similar to the original input data. We compared the 1,000 artificially generated images to original images located in the same class labels. Accordingly, some results were observably good, while there were some disrupted images as well. All these results were accomplished through the network parameters presented in Table 2.

Table 3. The example images obtained by DCGAN by using the corresponding original dataset images

SMIDS

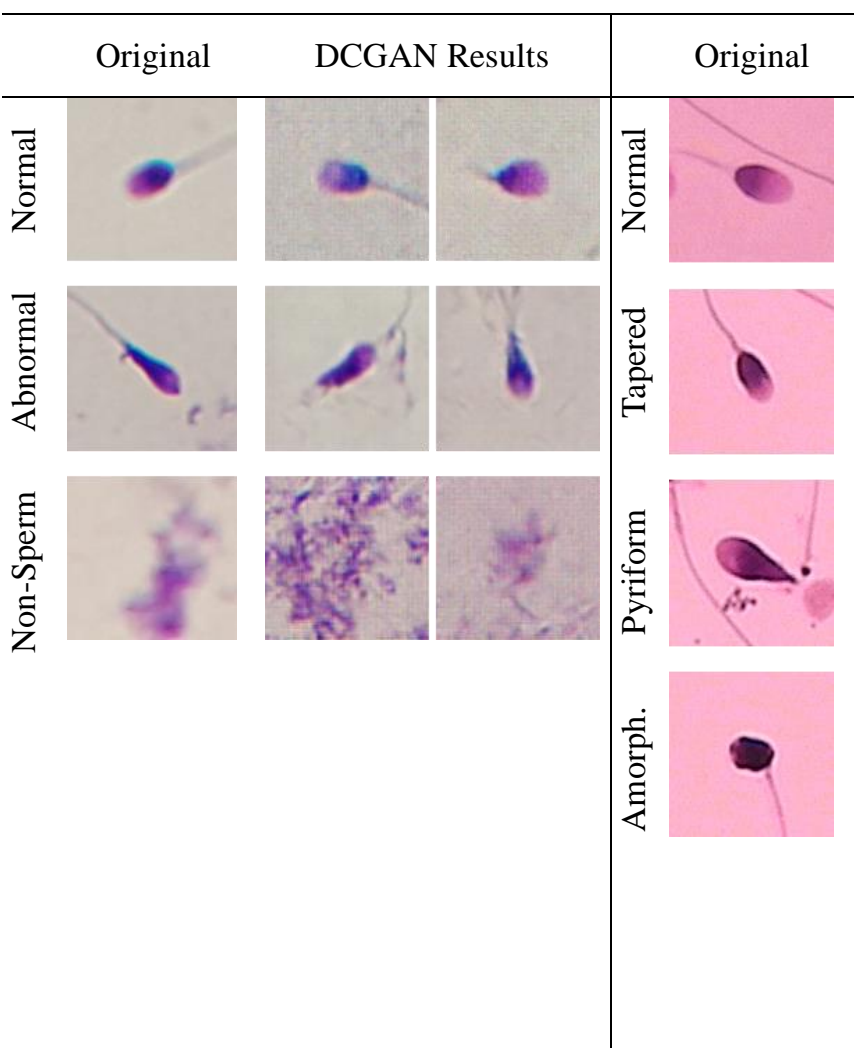

HuSHeM

SCIAN-Morpho

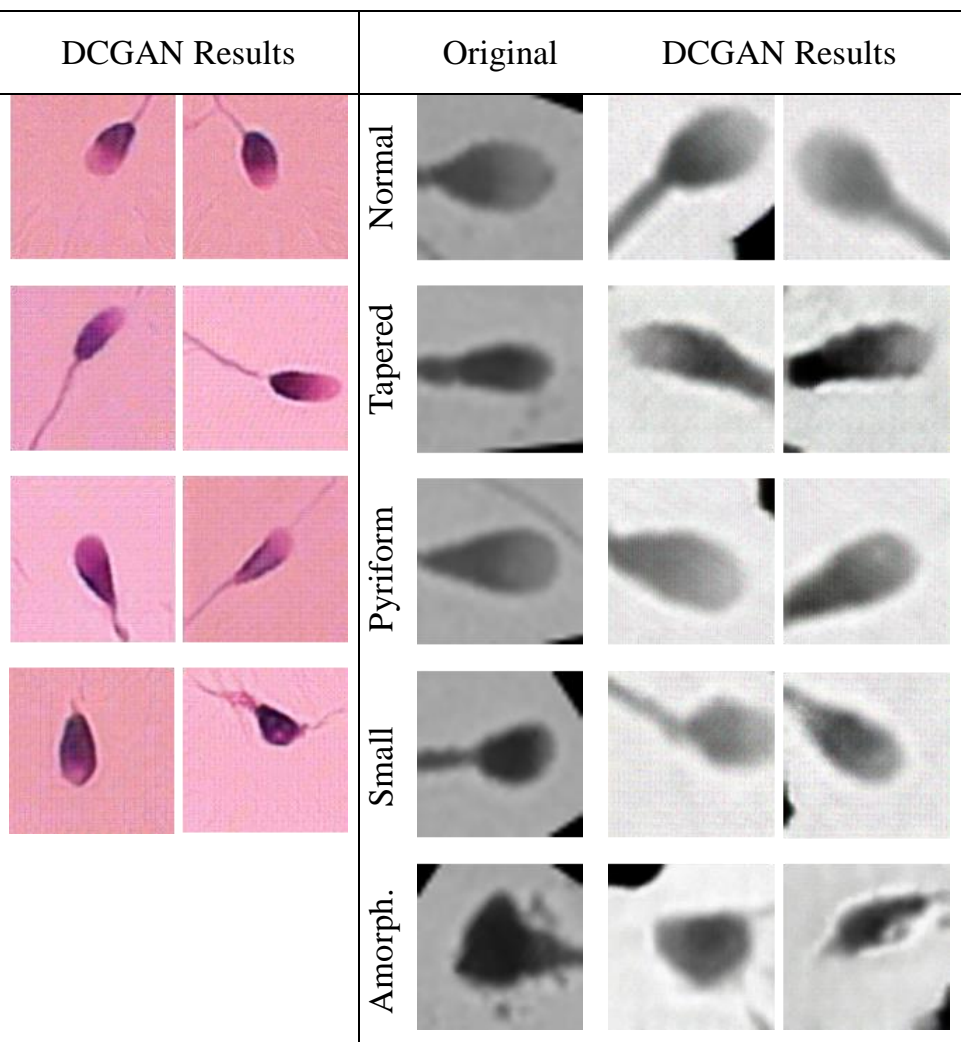

According to Figure 3, the best-generated images were obtained for the SMIDS dataset due to the lowest FID scores. An average of 29 FID score was measured for the SMIDS dataset. Images generated for the normal class were the most similar images to the original dataset. The less similar images have been generated for the Non-Sperm class; however, it is still better than e-ISSN: 2148-2683 other classes for HuSHeM and SCIAN-Morpho datasets. The reason for this performance for SMIDS is about the sample sizes of the original dataset. SMIDS has significantly more images than $\mathrm{HuSHeM}$ and SCIAN-Morpho datasets which provides a better training process for DCGAN. On the contrary, HuSHeM has the fewest sample size. Therefore, the FID score was 
measured as an average of 44.25. It is worse than SMIDS but better than SCIAN-Morpho due to having high-resolution images. The amorphous class is the most challenging class for HuSHeM because many abnormalities have been defined in the same class. Lastly, image generation for the SCIAN-Morpho dataset resulted in the worst FID scores due to having fewer samples, data imbalance, and low-resolution problems. An average of 53.46 FID score was measured for the image generation of the SCIAN-Morpho dataset.

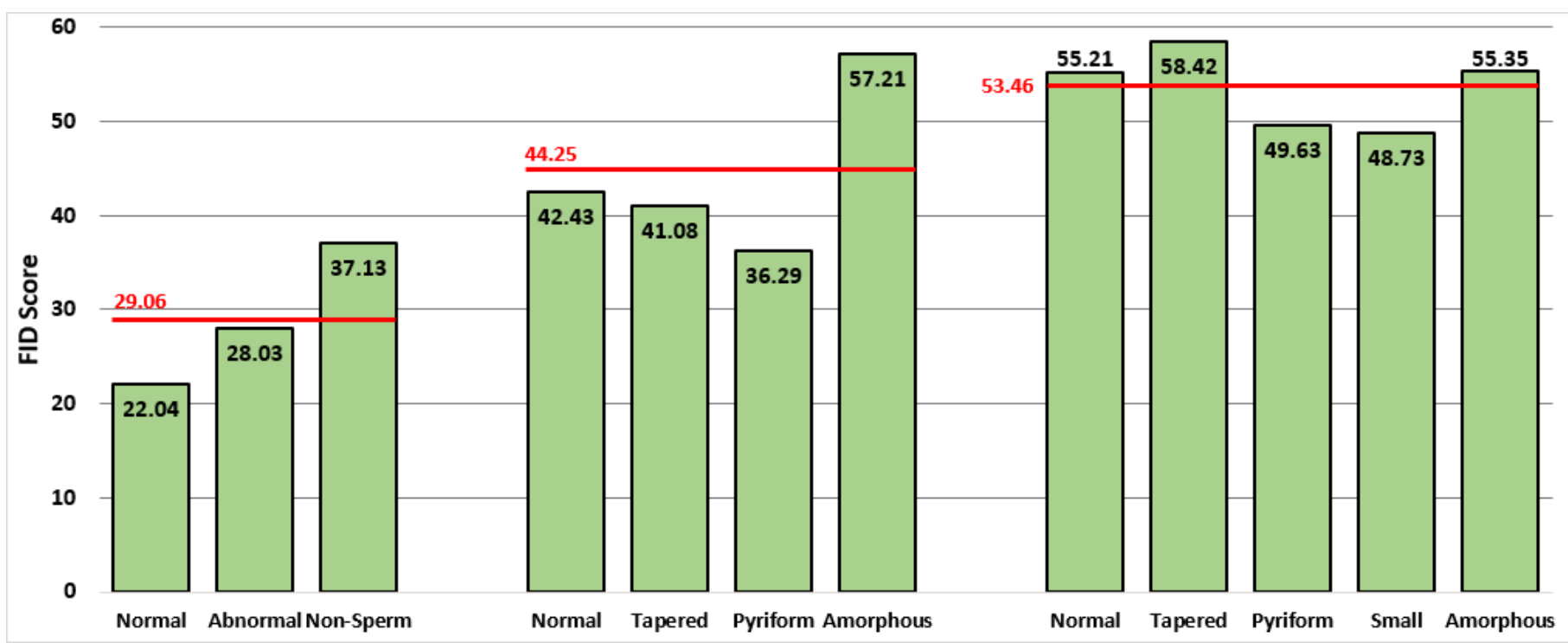

Figure 3. FID score over DCGAN-based generated datasets (Red Lines indicate the average FID scores per dataset)

\section{Conclusion}

In this study, one of the deep learning-based data augmentation techniques, DCGAN, has been implemented for the sperm morphology datasets in order to generate synthetic images. The performances of the DCGAN in terms of similarity between the generated and original images have been evaluated by FID scores. Additionally, an open-source GUI has been developed, in which the system can interact with the user for the fine-tuning procedure. DCGAN architecture has been used together with ReLU and Leaky ReLU activations, along with Adam optimization for high-resolution modeling. The proposed approach has been tested with three different sperm morphology datasets having different characteristics such as resolution, sample size, and color space. As an outcome of the comparisons, the best results have been inferred for the normal class of the SMIDS dataset with an FID score of 22.041, for the pyriform class of the HuSHeM dataset with an FID score of 36.297, and for the small class of SCIAN-MorphoSpermGS dataset with FID score 48.738. The results of this study indicated that one of the deep learning-based data augmentation techniques, DCGAN, is an effective technique to use as generating the synthetic sperm patch images for the augmentation. Adversarial networks can moderately recognize sensible characterizations of sperm shapes to form new images for generative modeling. While traditional spatial domain-based data augmentation techniques are effective in sperm morphological analysis, other techniques provided by GAN are promising as a preprocessing step of classification for future studies. We will also aim to use the created synthetic augmented datasets by DCGAN in the training of classification models such as inception, VGG16, etc., to enhance the classification performance in future studies.

\section{References}

Balayev, K., \& et al. (2020). Synthetic data generation with DCGAN. GitHub. https://github.com/Kamran017/SyntheticData-Generation-With-DCGAN

Barışkan, M. A., Orman, Z., \& Şamlı, R. (2020). Common generative adversarial network types and practical applications. Avrupa Bilim ve Teknoloji Dergisi, 585-590.

Chang, V., Garcia, A., Hitschfeld, N., \& Härtel, S. (2017). Goldstandard for computer-assisted morphological sperm analysis. Computers in Biology and Medicine, 83, 143-150.

Goodfellow, I., Pouget-Abadie, J., Mirza, M., Xu, B., WardeFarley, D., Ozair, S., Courville, A., \& Bengio, Y. (2014). Generative adversarial nets. In Z. Ghahramani, M. Welling, C. Cortes, N. Lawrence, \& K. Q. Weinberger (Eds.), Advances in Neural Information Processing Systems (27).

Ilhan, H. O, Sigirci, I. O., Serbes, G., \& Aydin, N. (2020). A fully automated hybrid human sperm detection and classification system based on mobile-net and the performance comparison with conventional methods. Medical \& Biological Engineering \& Computing, 58(5), 1047-1068.

Ilhan, H. O., \& Aydin, N. (2018). A novel data acquisition and analyzing approach to spermiogram tests. Biomedical Signal Processing and Control, 41, 129-139.

Kapoor, D. A. (2021). The changing landscape of urologic practice. Urologic Clinics, 48(2).

Karras, T., Aila, T., Laine, S., \& Lehtinen, J. (2018). Progressive growing of GANs for improved quality, stability, and variation. https://arxiv.org/abs/1710.10196

Karras, T., Laine, S., \& Aila, T. (2019). A style-based generator architecture for generative adversarial networks. 2019 IEEE/CVF Conference on Computer Vision and Pattern Recognition (CVPR), 4396-4405. 
Ledig, C., Theis, L., Huszár, F., Caballero, J., Cunningham, A., Acosta, A., Aitken, A., Tejani, A., Totz, J., Wang, Z., \& Shi, W. (2017). Photo-realistic single image super-resolution using a generative adversarial network. 2017 IEEE Conference on Computer Vision and Pattern Recognition (CVPR), 105-114.

Mirza, M., \& Osindero, S. (2014). Conditional generative adversarial nets. http://arxiv.org/abs/1411.1784

Radford, A., Metz, L., \& Chintala, S. (2016). Unsupervised representation learning with deep convolutional generative adversarial networks. https://arxiv.org/abs/1511.06434

Rubin, M., Stein, O., Giryes, R., Roitshtain, D., \& Shaked, N. T. (2018). Quantitative phase maps of live cells classified by transfer learning and generative adversarial network (GAN). Imaging and Applied Optics 2018 (3D, AO, AIO, COSI, DH, IS, LACSEA, LS\\&C, MATH, PCAOP), CW2E.7.

Rubin, M., Stein, O., Turko, N. A., Nygate, Y., Roitshtain, D., Karako, L., Barnea, I., Giryes, R., \& Shaked, N. T. (2019). TOP-GAN: Stain-free cancer cell classification using deep learning with a small training set. Medical Image Analysis, $57,176-185$.

Shaker, F., Monadjemi, S. A., Alirezaie, J., \& Naghsh-Nilchi, A. R. (2017). A dictionary learning approach for human sperm heads classification. Computers in Biology and Medicine, 91, 181-190.

Tortumlu, O. L., \& Ilhan, H. O. (2020). The analysis of mobile platform based CNN networks in the classification of sperm morphology. 2020 Medical Technologies Congress (TIPTEKNO), 1-4.

Vasconcelos, C. N., \& Vasconcelos, B. N. (2017). Increasing deep learning melanoma classification by classical and expert knowledge based image transforms. http://arxiv.org/abs/1702.07025

Yeh, R. A., Chen, C., Lim, T. Y., Schwing, A. G., HasegawaJohnson, M., \& Do, M. N. (2017). Semantic image inpainting with deep generative models. 2017 IEEE Conference on Computer Vision and Pattern Recognition (CVPR), 6882-6890.

Yüzkat, M., Ilhan, H. O., \& Aydın, N. (2020). Morphological classification of low quality sperm images using deep learning networks. 2020 Medical Technologies Congress (TIPTEKNO), 1-4.

Zhang, H., Xu, T., Li, H., Zhang, S., Wang, X., Huang, X., \& Metaxas, D. (2017). StackGAN: Text to photo-realistic image synthesis with stacked generative adversarial networks. 2017 IEEE International Conference on Computer Vision (ICCV), 5908-5916.

Zhao, J., Mathieu, M., \& LeCun, Y. (2017). Energy-based generative adversarial network. https://arxiv.org/abs/1609.03126

Zheng, Z., \& Liu, J. (2020). $P^{2}$-GAN: Efficient style transfer using single style image. https://arxiv.org/abs/2001.07466 